\title{
The role of tumor-associated macrophages in solid malignancies - an overview of current knowledge
}

\author{
Jozef Muria, Jaroslava Chylikovab ${ }^{b}$ Jozef Skardac, Maria Miklosovaa, Vojtech Kamarad ${ }^{b}$
}

Tumor-associated macrophages are an important part of the tumor microenvironment. The presence of certain populations of macrophages within tumor tissue may be associated with either better or worse disease prognosis. The study of these cells is currently receiving a great deal of attention, with the most important topics of investigation raised being: the typification of subpopulations of tumor-associated macrophages; identification of the prognostic significance of population density and distribution of macrophages in the tumor microenvironment; ways to influence macrophage activity, migration and differentiation within the tumor. The answers to these questions can improve the efficiency of immunoterapy for malignancies. The presented article briefly reviews recent findings on tumor-associated macrophages in solid malignancies.

Key words: tumor microenvironment (TME), tumor-associated macrophages (TAMs), resident tissue macrophages (NTAMs), classically activated macrophages (M1), alternatively activated macrophages (M2)

Received: September 16, 2021; Revised: September 16, 2021; Accepted: December 1, 2021; Available online: December 17, 2021 https://doi.org/10.5507/bp.2021.070

(c) 2022 The Authors; https://creativecommons.org/licenses/by/4.0/

${ }^{a}$ Department of Anatomy, Faculty of Medicine, University of Ostrava, Ostrava, Czech Republic

${ }^{b}$ Department of Histology and Embryology, Faculty of Medicine, University of Ostrava, Ostrava, Czech Republic

'Department of Pathology, Faculty of Medicine, University of Ostrava, Ostrava, Czech Republic and Department of Clinical and Molecular Pathology, Faculty of Medicine and Dentistry, Palacky University, Olomouc, Czech Republic

Corresponding author: Jaroslava Chylikova, e-mail: jaroslava.chylikova@osu.cz

\section{INTRODUCTION}

Malignant tumor tissue consists of tumor stroma and tumor parenchyma, which together form a tumor microenvironment (TME). The survival, growth and invasion of tumor parenchymal cells depend on their interactions with stromal cells, which consist of non-malignant cells of different cell types ${ }^{1-5}$.

Macrophages are an important part of the tumor microenvironment ${ }^{1-28}$; different populations of macrophages coexist within and around the tumor. In general terms, these are populations of tumor-associated macrophages (TAMs) as well as macrophages belonging to resident tissue macrophage, thus classified as non-tumor-associated macrophages (NTAMs). Garrido-Martin et al. report that TAMs in primary lung carcinomas have completely different transcriptional characteristics compared to NTAMs that are present in the surrounding non-tumor lung parenchyma of the same lung resection as in patients treated primarily surgically ${ }^{6}$. These findings demonstrate that TAMs undergo modifications as a result of the tumor tissue microenvironment ${ }^{2-4,6,8,10,12,13,19-24}$.

TAMs control and mediate many processes and interactions within the tumor, as well as interactions between the tumor and the host organism ${ }^{2-4,6,11}$. They enter the tumor tissue microenvironment primarily by migration and transformation of circulating monocytes ${ }^{4,6,13}$, although some subpopulations of macrophages arise from precursor cells independently of monocytes ${ }^{2,4,8,9,11}$.
It is well known that, in the tumor microenviroment, macrophages demonstrate ambivalent behavior as a result of their different differentiation ${ }^{2-4,6-8,10,12-15,18}$. Phenotypic diversity and functional plasticity are characteristic features of macrophages which allow them to perform a wide range of often conflicting tasks $2,4,6,8-10,12-15,17,18$. Important macrophages effector functions are phagocytosis, antigen presentation, secretion of variety signaling molecules. So they may affect antitumor immune response, angiogenesis and also inflamation.

Under the influence of various signals, macrophages can alter the range of expressed genes, the spectrum of surface membrane molecules and the production of metabolites and mediators. We are talking about the polarization of macrophages ${ }^{2,8,11,12,17,18,29}$. Classically activated macrophages are pro-inflammatory and have an inhibitory effect on the tumor; they are referred to as M1 macrophages. Alternatively activated macrophages are anti-inflammatory and they promote the persistence, or progression, of the tumor respectively. They are referred to as M2 macrophages s. $^{2-4,6,8,10,13-15,19,30}$.

Recent studies point to the pitfalls of the rigid division of TAMs into the classes of M1 and M2 macrophages $^{6,29-31}$. Traits of the M1 and M2 phenotypes are not mutually exclusive, even in a particular cell ${ }^{6,8,16,20,30}$. Garrido-Martin et al. demonstrated that in some patients with primary lung adenocarcinomas and squamous-cell carcinomas, a portion of M2 macrophages showed strong simultaneous expression of M1 markers (M1hotTAMs), 
these were identified using CXCL9. The presence of M1hotTAMs in the tissue microenvironment of primary lung carcinoma was associated with intense tumor infiltration by resident cytotoxic tissue memory cells CD8 + TRM and with concomitant better survival ${ }^{6}$. Although the mechanisms leading to $\mathrm{T}$ cell colonization of the tumor are only partially known, M1hotTAMs play an important role ${ }^{6,30}$. They have a high expression of CXCL9, CXCL10, CXCL11 and CXCL12; these molecules are potent chemoattractants and activators of $T$ cells. The presence of M1hotTAMs in the tumor is associated with a higher rate of CD8 + and CXCR3 + T cell infiltration. Higher population density of tumor infiltrating $\mathrm{T}$ cells within non-small cell lung carcinoma is associated with a better prognosis of the disease. M1hotTAMs also express a large number of surface antigen presenting molecules. The high rate of presentation of tumor antigens contributes to the maintenance of $\mathrm{T}$ cell infiltration of the tumor ${ }^{30}$.

Therefore, expression of M2 markers by macrophages does not necessarily guarantee their immunosuppressive action. Patients with primary lung adenocarcinoma infiltrated with M1hotTAMs had better survival rates regardless of concomitant coexpression of M2 markers ${ }^{6}$.

M1 and M2 TAMs represent heterogeneous groups of cells that can be further subtyped into subgroups with dramatically different transcriptomes ${ }^{6,8}$.

The population density of macrophages in the tumor microenvironment, as well as their phenotypic and functional differentiation, is influenced by various factors. The tumor produces TDCF (tumor-derived chemotactic factor), which is identical to CCL2 (C-C motif chemokine ligand 2) and increases the migration of monocytes into the tumor ${ }^{17}$. Tumor cell secretion affects the transcriptional profile of macrophages and their polarization, leading to shifts in the balance between M1 and M2 features of macrophages in the tumor tissue ${ }^{10,17,20}$. Balance between M1 and M2 macrophages may by influenced by oxygen availability in tumor parenchyma, or by variety of stress conditions such as endoplasmic reticular stress, oxidative stress, osmotic stress and also cytokine stimulation ${ }^{20}$.

Apoptosis and necrosis are important events in the tumor microenvironment. Tumor cell apoptosis promotes M2 and suppresses M1 polarization ${ }^{24}$. At the molecular level, S1P (sphingosine-1-phosphate) and microRNA-375 are possible factors derived from apoptotic cells that influence the polarization of macrophages ${ }^{22,23}$.

The effect of necrosis is probably complex. Reiter et al. report the potentiation of the antitumor properties of macrophages by necrotic cells through increased NO production. Other studies highlight the absence of inflammatory cytokine production following phagocytosis of necrotic tumor fragments and increased infiltration of necrotic tumor sites by macrophages. These events support tumor progression ${ }^{12}$.

Hypoxia in the tumor microenvironment is associated with the production of hypoxia-induced chemotactic factors. Accumulation of M2 macrophages in hypoxic areas of advanced tumors is known as an angiogenesis promoting factor ${ }^{4,17,19,20,31}$.
The tumor represents a heterogeneous tissue structure. Individual compartments of the tumor microenvironment are preferentially populated by macrophages with different cellular phenotypes and biological properties ${ }^{10,17}$. The representation and distribution of TAMs in a tumor are related to its biological properties, and thus to the course of the tumor ${ }^{10,13,25,26}$.

\section{Interaction of TAMs with tumor}

TAMs can promote tumor parenchyma proliferation, stimulate tumor angiogenesis, contribute to the metastatic process, reduce the availability of essential molecules for other immunocytes, as well as modulate the T cell-mediated anti-tumor immune response $\mathrm{e}^{2-4,6-8,10,14,21,31}$.

The presence of M1hotTAMs in the tumor microenvironment is accompanied by an increased density of tumor infiltrating $\mathrm{T}$ cells. In contrast, a high concentration of M2 macrophages, in the absence of M1 markers, is associated with a low density of T cells ${ }^{6}$.

Thus, the gene expression profile and polarization of macrophages in the tumor affect $\mathrm{T}$ cell-mediated antitumor immunity $6,15,26,30,31$.

The broad spectrum of cytokines produced by macrophages exhibits antagonistic effects relative to the tumor. IL-10, IFN- $\gamma$ and TNF- $\alpha$ modulate the local inflammatory response and also the systemic immune response ${ }^{10}$.

IL-10, through its anti-inflammatory and immunosuppressive action, favors tumor escape from immune surveillance ${ }^{10,12,31}$. Contrary to some authors that report the immunostimulatory effects of IL-10 ${ }^{10}$.

TNF- $\alpha$ contributes to the maintenance of chronic inflammation in the tumor microenvironment, supporting the growth of tumor cells and also their metastatic potential. Furthermore, it also contributes to the activation of NK cells and CD $8+\mathrm{T}$ cells, thereby strengthening and maintaining the body's antitumor response. TNF- $\alpha$ also influences tumor cells by directly inducing apoptosis ${ }^{10,12}$.

On one hand, IFN- $\gamma$ promotes the proliferation of tumor cells, inhibits their recognition by NK cells and blocks apoptosis. On the other hand, IFN- $\gamma$ may inhibit tumor angiogenesis ${ }^{10}$.

Interpretation of the role of these cytokines, as well as many others, in the evolution of the tumor as a whole is complex. Some studies suggest the absence of any association between the levels of these cytokines and the prognosis of patients with NSCLC.

\section{Macrophages as a potential target for treatment strategies}

Many studies point to a possible link between macrophages and cancer treatment failure ${ }^{15,17,18,28,31}$. This emphasizes the need to focus on macrophages as a potential target for new therapeutic approaches.

Increased CCL2 production, in doxorubicin-treated breast cancer, led to the accumulation of CCR2 + monocytes in the tumor, which contributed to the relapse of the disease. A similar finding has been found in some types of colorectal cancer treated with 5 -fluorouracil ${ }^{17}$.

Tumor-associated macrophages may limit the effect of treatment based on immune checkpoint inhibitors 
such as atezolizumab, durvalumab or avelumab, and also new PD-L1 inhibitors in clinical developments ${ }^{17,18,31}$, although there are likely more numerous mechanisms at play. TAMs express a wide variety of inhibitory immune control checkpoint ligands that are different from those to which immunotherapy is directed. These ligands can induce a state of strong immunosuppression. TAMs can bind a therapeutically administered antibody to its Fc receptor, several times more intensely and for a longer duration than the receptor of the target $\mathrm{T}$ cell. By this binding, they weaken the therapeutic effect of the used antibody ${ }^{17}$.

Angiogenesis inhibitors slow the growth of a tumor by compromising its adequate supply of nutrients and remediation of the products of its metabolism. During treatment with angiogenesis inhibitors, hypoxia is accentuated in the tumor tissue. Hypoxia potentiates the chemoattraction of macrophages into the tumor microenvironment, which in turn produces growth factors, such as VEGF, TNF- $\alpha$, IL-1 $\beta$, IL-8 (CXCL8), platelet-derived growth factor (PDGF), basic fibroblast growth factor (bFGF), thymidine phosphorylase, MMPs and other molecules important for angiogenesis ${ }^{4,7,19,20,31}$. The high density of TAMs has been evaluated, by several studies, as a poor prognostic factor in relation to treatment with angiogenesis inhibitors ${ }^{17}$. The logical conclusion is that the reduction in the density of TAMs, respective to the density of some of their populations, in the tumor microenvironment may potentiate the effect of treatment with angiogenesis inhibitors. Dalton et al. reported an improvement in the effect of anti-VEGF monoclonal antibody treatment by zolendronic acid-induced depletion of TAMs in several ovarian cancer tumor models ${ }^{32}$.

\section{CONCLUSION}

Macrophages are an important part of the tumor microenvironment. They are characterized by great phenotypic diversity, which predetermines their diverse functions.

Depending on the cell phenotype and functional differentiation, macrophages may potentiate or suppress tumor growth. Investigation of the role of individual populations of macrophages in the development of cancer illness depends on the accuracy of their identification. Expression of a particular trait need not be exclusive and preclude co-expression of the traits of others.

Tumor stromal cells are genetically stable compared to tumor parenchymal cells. They could thus be a beneficial therapeutic target, with more predictable behavior.

Influencing the activity, migration and differentiation of macrophages, due to their key role in the tangle of cell interactions, can be reflected in the overall effectiveness of anticancer treatment. Macrophages thus represent a hope for increasing the effectiveness of existing treatment modalities and possibly for the development of completely new methods in the treatment of malignant diseases.
Author contributions: JM: literature search, manuscript writing; JCH: manuscript writing; MM: critical revision; JS: critical revision; VK: concept, critical revision.

Conflict of interest statement: The authors state that there are no conflicts of interest regarding the publication of this article.

\section{REFERENCES:}

1. Burdova A, Rulisek P, Bouchal J, Král M, Student V, Kolar Z. Infiltration of prostate cancer by CD204+ and CD3+ cells correlates with ERG expression and TMPRSS2-ERG gene fusion. Klin Onkol 2018;31(6):42128.

2. Kielbassa K, Vegna S, Ramirez C, Akkari L. Understanding the origin and diversity of macrophages to tailor their targeting in solid cancers. Front Immunol 2019;10:2215.

3. Chen Y, Zhang S, Wang Q, Zhang X. Tumor-recruited M2 macrophages promote gastric and breast cancer metastasis via M2 macrophage-secreted CHI3L1 protein. J Hematol Oncol 2017;10(1):36.

4. Yang L, Zhang Y. Tumor-associated macrophages: from basic research to clinical application. J Hematol Oncol 2017;10(1):58.

5. Teng F, Tian WY, Wang YM, Zhang YF, Guo F, Zhao J, Gao C, Xue FX. Cancer-associated fibroblasts promote the progression of endometrial cancer via the SDF-1/CXCR4 axis. J Hematol Oncol 2016;9:8.

6. Garrido-Martin EM, Mellows TWP, Clarke J, Ganesan AP, Wood O, Cazaly A, Seumois G, Chee SJ, Alzetani A, King EV, Hedrick CC, Thomas G, Friedmann PS, Ottensmeier CH, Vijayanand P, SanchezElsner T. M1 ${ }^{\text {hot }}$ tumor-associated macrophages boost tissue-resident memory T cells infiltration and survival in human lung cancer. J Immunother Cancer 2020;8(2):e000778.

7. Cassetta L, Fragkogianni S, Sims AH, Swierczak A, Forrester LM, Zhang $\mathrm{H}$, Soong DYH, Cotechini T, Anur P, Lin EY, Fidanza A, LopezYrigoyen M, Millar MR, Urman A, Ai Z, Spellman PT, Hwang ES, Dixon JM, Wiechmann L, Coussens LM, Smith HO, Pollard JW. Human tumor-associated macrophage and monocyte transcriptional landscapes reveal cancer-specific reprogramming, biomarkers, and therapeutic targets. Cancer Cell 2019;35(4):588-602.e10.

8. Quail DF, Joyce JA. Molecular pathways: deciphering mechanisms of resistance to macrophage-targeted therapies. Clin Cancer Res 2017;23(4):876-84.

9. Gordon S, Plüddemann A. The mononuclear phagocytic system. Generation of diversity. Front Immunol 2019;10:1893.

10. Jackute J, Zemaitis M, Pranys D, Sitkauskiene B, Miliauskas S, Vaitkiene S, Sakalauskas R. Distribution of M1 and M2 macrophages in tumor islets and stroma in relation to prognosis of non-small cell lung cancer. BMC Immunol 2018;19(1):3.

11. Loyher $\mathrm{PL}$, Hamon $\mathrm{P}$, Laviron $M$, Meghraoui-Kheddar A, Goncalves E, Deng Z, Torstensson S, Bercovici N, Baudesson de Chanville C, Combadière B, Geissmann F, Savina A, Combadière C, Boissonnas A. Macrophages of distinct origins contribute to tumor development in the lung. J Exp Med 2018;215(10):2536-53.

12. Sarode P, Schaefer MB, Grimminger F, Seeger W, Savai R. Macrophage and tumor cell cross-talk is fundamental for lung tumor progression: we need to talk. Front Oncol 2020;10:324.

13. Sumitomo R, Hirai T, Fujita M, Murakami H, Otake $Y$, Huang CL. M2 tumor-associated macrophages promote tumor progression in nonsmall-cell lung cancer. Exp Ther Med 2019;18(6):4490-98.

14. Hwang I, Kim JW, Ylaya K, Chung EJ, Kitano H, Perry C, Hanaoka J, Fukuoka J, Chung JY, Hewitt SM. Tumor-associated macrophage, angiogenesis and lymphangiogenesis markers predict prognosis of non-small cell lung cancer patients. J Transl Med 2020;18(1):443.

15. Ngambenjawong C, Gustafson HH, Pun SH. Progress in tumor-associated macrophage (TAM)-targeted therapeutics. Adv Drug Deliv Rev 2017;114:206-21.

16. Singhal S, Stadanlick J, Annunziata MJ, Rao AS, Bhojnagarwala PS, O'Brien S, Moon EK, Cantu E, Danet-Desnoyers G, Ra HJ, Litzky L, Akimova T, Beier UH, HancockWW, Albelda SM, Eruslanov EB. Human tumor-associated monocytes/macrophages and their regulation of T cell responses in early-stage lung cancer. Sci Transl Med 2019;11(479):eaat1500. 
17. Anfray C, Ummarino A, Andón FT, Allavena P. Current strategies to target tumor-associated-macrophages to improve anti-tumor immune responses. Cells 2019;9(1):46.

18. Mantovani A, Allavena P. The interaction of anticancer therapies with tumor-associated macrophages. J Exp Med 2015;212(4):435-45.

19. Yang M, McKay D, Pollard JW, Lewis CE. Diverse functions of macrophages in different tumor microenvironments. Cancer Res 2018;78(19):5492-503

20. Wenes M, Shang M, Di Matteo M, Goveia J, Martín-Pérez R, Serneels $\mathrm{J}$, Prenen $\mathrm{H}$, Ghesquière $\mathrm{B}$, Carmeliet $\mathrm{P}$, Mazzone M. Macrophage metabolism controls tumor blood vessel morphogenesis and metastasis. Cell Metab 2016;24(5):701-15.

21. Arwert EN, Harney AS, Entenberg D, Wang Y, Sahai E, Pollard JW, Condeelis JS. A unidirectional transition from migratory to perivascular macrophage is required for tumor cell intravasation. Cell Rep 2018;23(5):1239-48.

22. Frank AC, Ebersberger S, Fink AF, Lampe $S$, Weigert $A$, Schmid $T$, Ebersberger I, Syed SN, Brüne B. Apoptotic tumor cell-derived microRNA-375 uses CD36 to alter the tumor-associated macrophage phenotype. Nat Commun 2019;10(1):1135.

23. Syed SN, Weigert A, Brüne B. Sphingosine kinases are involved in macrophage NLRP3 inflammasome transcriptional induction. Int J Mol Sci 2020;21(13):4733.

24. Voss JJLP, Ford CA, Petrova S, Melville L, Paterson M, Pound JD, Holland P, Giotti B, Freeman TC, Gregory CD. Modulation of macrophage antitumor potential by apoptotic lymphoma cells. Cell Death Differ 2017;24(6):971-83.

25. Wu P, Wu D, Zhao L, Huang L, Chen G, Shen G, Huang J, Chai Y. Inverse role of distinct subsets and distribution of macrophage in lung cancer prognosis: a meta-analysis. Oncotarget 2016;7(26):40451-60.
26. Mei J, Xiao Z, Guo C, Pu Q, Ma L, Liu C, Lin F, Liao H, You Z, Liu L. Prognostic impact of tumor-associated macrophage infiltration in non-small cell lung cancer: a systemic review and meta-analysis. Oncotarget 2016;7(23):34217-28.

27. Caux C, Ramos RN, Prendergast GC, Bendriss-Vermare N, MénétrierCaux C. A milestone review on how macrophages affect tumor growth. Cancer Res 2016;76(22):6439-42.

28. Mantovani A, Marchesi F, Malesci A, Laghi L, Allavena P. Tumourassociated macrophages as treatment targets in oncology. Nat Rev Clin Oncol 2017;14(7):399-416.

29. Yáñez A, Coetzee SG, Olsson A, Muench DE, Berman BP, Hazelett DJ, Salomonis N, Grimes HL, Goodridge HS. Granulocyte-monocyte progenitors and monocyte-dendritic cell progenitors independently produce functionally distinct monocytes. Immunity 2017;47(5):890902.e4.

30. van Dalen FJ, van Stevendaal MHME, Fennemann FL, Verdoes M, Ilina O. Molecular repolarisation of tumour-associated macrophages. Molecules 2018;24(1):9.

31. Xiang X, Wang J, Lu D, Xu X. Targeting tumor-associated macrophages to synergize tumor immunotherapy. Signal Transduct Target Ther 2021;6(1):75.

32. Dalton HJ, Pradeep S, McGuire M, Hailemichael $Y$, Ma S, Lyons $Y$, Armaiz-Pena GN, Previs RA, Hansen JM, Rupaimoole R, GonzalezVillasana V, Cho MS, Wu SY, Mangala LS, Jennings NB, Hu W, Langley R, Mu H, Andreeff M, Bar-Eli M, Overwijk W, Ram P, Lopez-Berestein G, Coleman RL, Sood AK. Macrophages facilitate resistance to anti-VEGF therapy by altered VEGFR expression. Clin Cancer Res 2017;23(22):7034-46. 